\title{
Localization and Capacitance Fluctuations in Disordered Au Nano-junctions
}

\author{
M. Bowman, A. Anaya, A. L. Korotkov, and D. Davidović \\ Georgia Institute of Technology, Atlanta, GA 30332
}

(Dated: November 9, 2018)

\begin{abstract}
Nano-junctions, containing atomic-scale gold contacts between strongly disordered leads, exhibit different transport properties at room temperature and at low temperature. At room temperature, the nano-junctions exhibit conductance quantization effects. At low temperatures, the contacts exhibit Coulomb-Blockade. We show that the differences between the room-temperature and low temperature properties arise from the localization of electronic states in the leads. The charging energy and capacitance of the nano-junctions exhibit strong fluctuations with applied magnetic field at low temperature, as predicted theoretically.
\end{abstract}




\section{INTRODUCTION}

The prospect of molecular electronics as a potential alternative to conventional siliconbased electronics has lead to an increased interest in fabrication of atomic scale gaps and atomic-scale contacts between metallic electrodes. Examples include atomic-scale gaps formed by mechanically controlled break junctions, ${ }_{1.2}^{1.2}$ electrodeposition,, 3.4 .5 .6 .7 and electromigration ${ }^{8.9 .10}$. In these fabrication techniques, one can determine whether a junction has atomic-scale dimensions by changing the conductance of the junction around the conductance quantum $G_{Q}=e^{2} / h$. Discrete steps in conductance of order $G_{Q}$ indicate that the contacts have atomic scale dimensions. This scheme works remarkably well in cases where the gaps and the contacts are formed in ultra-high-vacuum (UHV) conditions, such as mechanically controlled break junctions at cryogenic temperatures. ${ }^{2}$

Some schemes for generating atomic-scale gaps involve exposure of these gaps to nonUHV environment, such as air ${ }^{8.10}$ or ionic solutions ${ }^{3.4 .5 .6 .7}$. In this case, intermixing between atoms in the leads and impurity molecules ( such as $\mathrm{H}_{2} \mathrm{O}$ ) can degrade the quality of the gaps. Understanding of electrical conduction in such disordered atomic-scale gaps and atomic-scale contacts is still lacking.

Recently, Yu and Natelson have studied Au nano-junctions formed by electroplating from an aqueous solution. .7 Transport measurements were carried out at both room temperature and cryogenic temperatures. The authors found different transport properties at room and low temperature. At room temperature, as the gap size between two Au leads is reduced by electroplating, conductance increased in discrete steps of order $G_{Q}$, suggesting that the contacts were atomic scale, consistent with the prior work. ${ }^{3.4 .5}$ In addition, the nano-junctions were Ohmic at room temperature.

At $T=1.8 K$, however, Au junctions with room temperature conductance $G(300 K) \sim$ $G_{Q}$, the conductance at zero bias voltage and $T=1.8 K$ was suppressed by $\approx 100 \%$, which was referred to as the zero-bias anomaly (ZBA). They argued that ZBAs displayed a suppression of the density of states in the leads at the Fermi level, as a result of disorder introduced by the electroplating process. The disorder was attributed to the grain boundaries and adsorption of impurities from the solution.

We have reported similar observations in Au nano-junctions formed by an electric-fieldinduced migration process. ${ }^{10}$ At room temperature, as the conductance of the junctions 

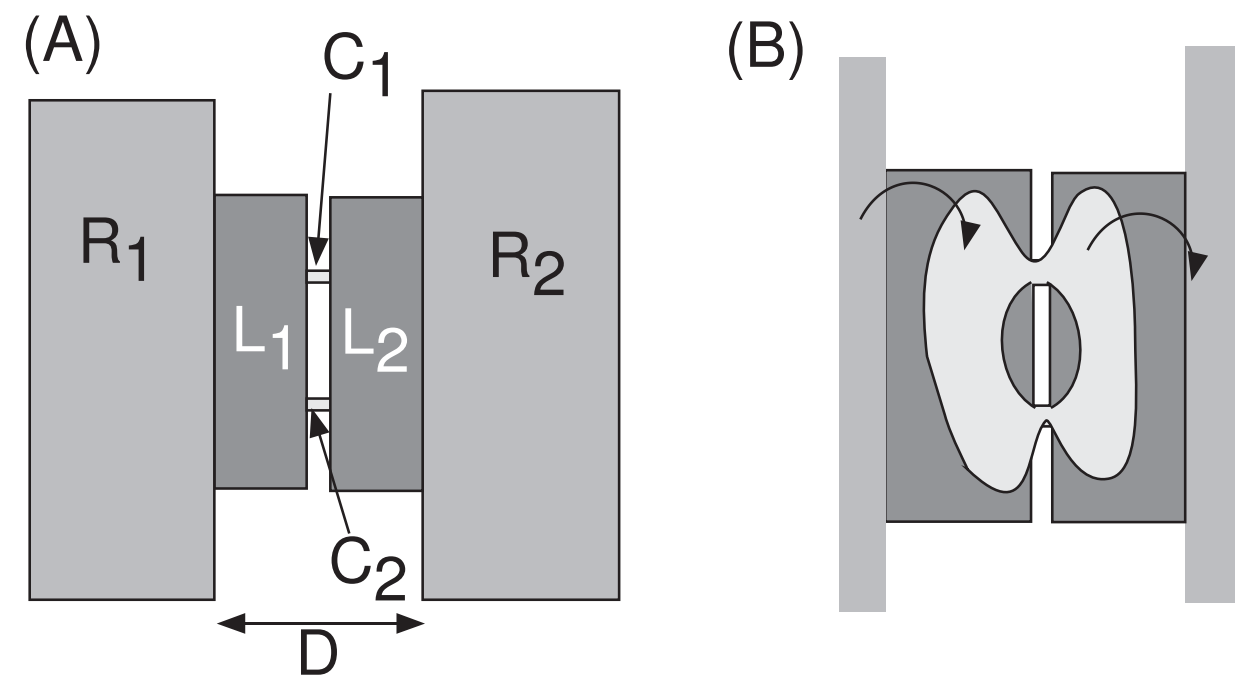

FIG. 1: A. Disordered Au nano-junction. B. Sequential electron tunnelling through the nanojunction, via a localized puddle of electrons.

increased from a value below the conductance quantum to above the conductance quantum, the conductance displayed discrete steps in conductance, of order $G_{Q}$. In addition, the room temperature I-V curves of the samples were linear (Ohmic).

At low temperatures, we found strong ZBAs in samples with $G(300 K) \sim G_{Q}$, similar to the ZBAs in electroplated Au nano-junctions. However, samples with $G(300 K)<G_{Q}$ were found to exhibit Coulomb blockade, proved by the quasiperiodic gate-voltage dependence of the conductance at $T=0.015 \mathrm{~K}$. Coulomb blockade was attributed to single-electron charging effects on one or a few grains in the leads. The data fit exceptionally well the theories of Coulomb Blockade in the weak ${ }^{11.12}$ and the strong coupling regimes ${ }^{13}$.

In this paper, we first show that Coulomb blockade in Au nano-junctions is not restricted to single electron charging on one or few metallic grains. In fact, Coulomb blockade is observed when the resistance of the leads is comparable with the resistance of the contacts, even if there are no apparent grains in the leads. We propose a general model of a disordered Au nano-junction containing atomic-scale contacts, which is sketched in Fig. 1. Reservoirs $R_{1}$ and $R_{2}$ are bulk $\mathrm{Au}$ films, which are good metals. $C_{1}$ and $C_{2}$ are atomic-scale metallic contacts that are responsible for conductance quantization at room temperature. $L_{1}$ and $L_{2}$ are highly disordered leads, with room temperature resistances smaller than or comparable to the resistance of atomic-scale contacts.

The model reconciles the difference between room and low temperature transport prop- 
erties of Au nano-junctions, as follows. The resistivity of the leads is assumed high enough to cause strong localization. However, the characteristic temperature at which localization suppresses conductivity in the leads is assumed to be smaller than $300 K$. In this case, the resistance of the contacts dominates at room temperature, explaining conductance quantization and Ohmic properties. At low temperatures, however, the resistance of the leads becomes much larger than the resistance of the atomic scale contacts, explaining CoulombBlockade and ZBAs. This interpretation of ZBAs in terms of localization is different from the alternative interpretation in terms of suppression of the density of states in the leads. ${ }^{6.7}$ In sec. $\nabla$ we explain the difference in more detail.

After our model is presented, we discuss capacitance fluctuations of the nano-junctions with applied magnetic field. The capacitance fluctuations in coherent conductors in the charging regime have been predicted theoretically, 14 but have not yet been demonstrated experimentally. The strong disorder combined with the small size of our nano-junctions makes it possible to study charging effects in the phase coherent regime, permitting us to demonstrate and explore capacitance fluctuations.

The paper is organized as follows: In section II we give a detailed summary of the nanojunction fabrication process and arrive at the nano-junction model shown in Fig. 1. A. In section [II we present Coulomb-blockade measurements and discuss electron localization in the leads. In section $[\mathrm{IV}$ we discuss capacitance fluctuations. In section $\square$ we explain the differences between our samples and electroplated nano-junctions.

\section{FABRICATION OF GOLD NANO-JUNCTIONS}

The fabrication of Au nano-junctions used in this paper has been described in Refs. ${ }^{10.15}$. In this section we summarize the fabrication process. We present new data and new images of the nano-junctions, which have improved our understanding of nano-junction properties since prior publications.

To create a nano-junction between two Au films, we deposit Au atoms over a 70nm wide slit, as shown in Fig. 2-A. The slit is created in $S i_{3} N_{4}$ using electron-beam lithography and etching. ${ }^{10}$ The large undercut serves to prevent the connection between two Au films. The exposed length of the slit is $0.1 \mathrm{~mm}$. The current between the films is monitored in situ. The current limiting resistor $R_{S}$ is added in series with the sample. 


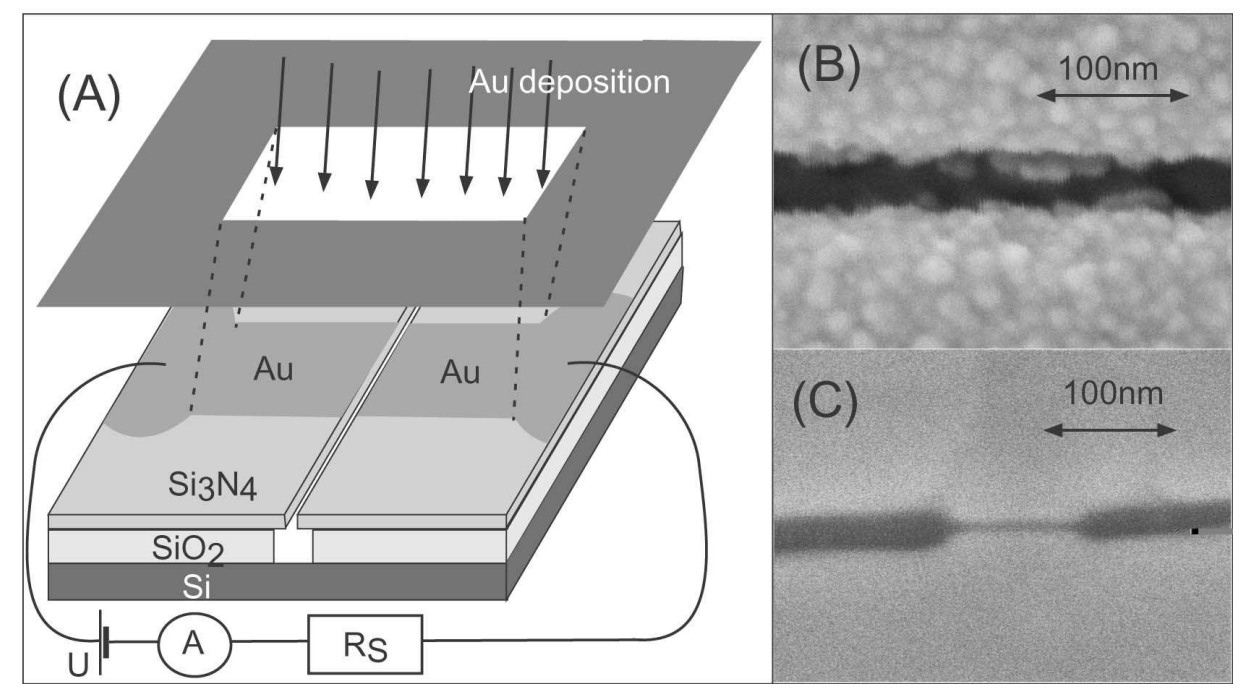

FIG. 2: A. Deposition of Au over a 70nm wide slit. B. Image of the gap between two $70 \mathrm{~nm}$ thick $\mathrm{Au}$ films grown at low bias voltage. C. Image of the gap between two $70 \mathrm{~nm}$ thick Au films grown at high bias voltage.

Gold deposition is done by thermal evaporation and the deposition rate is $2.5 \AA / s$. The background pressure of the deposition chamber measured near the gate valve of the pump is $\sim 10^{-7}$ Torr. Because water molecules outgas from the mask and other nearby surfaces, the sample pressure is higher. The pressure measured with a gauge placed near the sample is in the $10^{-6}$ Torr range.

During the deposition, the gap between two gold films is reduced in proportion to the film thickness. If the bias voltage is weak $(<0.1 \mathrm{~V})$, then the two gold films electrically connect when the thickness of the film reaches about 70nm. Fig. 2. B shows the shape of the gap between two $\mathrm{Au}$ films of thickness $70 \mathrm{~nm}$ grown at $U=0.1 \mathrm{~V}$. The films are not connected in this sample. The edge of the film is quite rough because of grains sticking to the edge of the gap. At $70 \mathrm{~nm}$ thickness, there is a $\sim 50 \%$ chance that there is a pair of grains attached on the opposite sides of the gap and that are in electric contact. By stoping the deposition at the moment when the desired current is detected, we create an atomic scale gap or an atomic scale contact. 


\section{A. Electric field induced surface diffusion}

The bias voltage has a strong influence on the shape and electric properties of the nanojunctions. In general, polarization effects from the applied electric field can induce atom migration processes with a "hierarchy of activation energies" 16 These processes include electric-field induced surface diffusion, migration due to localized heating, elastic and plastic deformation, and field desorption. The activation energy of these processes depends on both the electric field and the electric field gradient. It has been demonstrated that surface atom diffusion caused by the field gradient has the lowest activation energy: 16

In our samples, if the voltage applied between the films is large $(\sim 10 \mathrm{~V})$, a strong electric field inside the gap can pull a pair of protrusions from the opposing sides of the gap. Fig. 2-C shows the shape of the gap between two Au films grown at 20V. The edges of the films are much smoother than those in Fig. 2-B. In addition, the film, in the vicinity of the gap in Fig. 2.C, is also much smoother than the film in Fig. 2.-B.

These differences can be explained by field induced surface diffusion. At large bias voltage, roughness along the film edges (Fig. 2-B) induces field gradients, decreasing the activation energy for surface diffusion. In response, surface Au atoms diffuse where the electric field gradient is the strongest, thereby reducing surface roughness.

In the sample in Fig. 2-C, there is neither mechanical nor electric contact between the two films. This shows that the protrusion stopped growing on its own, before a contact could have established. The two protrusions are almost mirror images of each other.

Processes such as elastic and plastic deformation and field desorption are driven by the magnitude of the electric field, not the field gradient, and therefore can not be responsible for protrusion growth. The electric field in Fig. 2-C is strongest where the gap is smallest, which would increase the speed of the protrusion growth due to elastic or plastic deformation. On the other hand, the electric field gradient is weak inside this region, thereby decreasing the speed of protrusion growth due to surface diffusion.

\section{B. Tunnelling Contacts}

For most of our samples, the electric field induced surface diffusion leads to a contact between the two protrusions. Fig. 3-A and B show two such contacts, formed during growth 


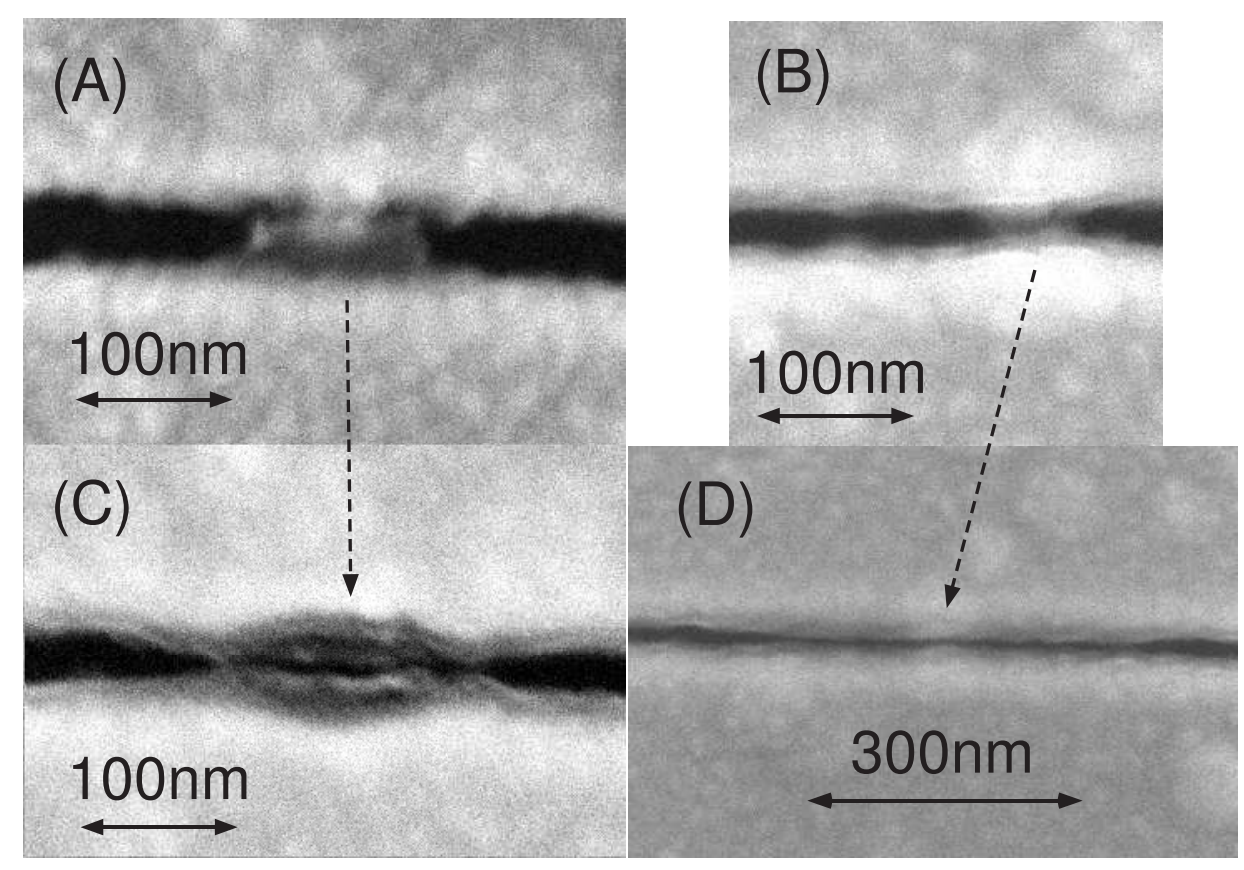

FIG. 3: A and B: Two tunnelling junctions formed at 10V bias voltage C and D: The same contacts, after the conductance is increased above $2 e^{2} / h$.

at 10V. Deposition of Au was stopped as soon as the slightest electric contact was detected. The electric contact was exposed to $10 \mathrm{~V}$ for $\approx 1$ s, and then the bias voltage was quickly reduced to zero (at a rate of $1 \mathrm{~V}$ in $10 \mathrm{~ms}$ ). I-V curves were obtained by measuring current while bias voltage was reduced to zero. The samples were subsequently transferred to the scanning electron microscope (SEM) and images were taken.

The resistance of the junctions is large compared to the resistance quantum. The I-V curves fit quite well the model of field emission through a tunnelling barrier with a barrier height close to the work function of $\mathrm{Au}(5.1 \mathrm{eV})$ and the barrier thickness of about $10 \AA$, as shown in Fig. 44. The fitting is described in Ref. 15

The key point that we want to make here is that the voltage drop of $10 \mathrm{~V}$ is not distributed uniformly through the leads. It is localized within a single tunnelling junction. If it were otherwise, the I-V curve would exhibit less barrier bending than that in Fig. 4. A. For example, assume that there are two tunnelling junctions with the same resistance and barrier height, connected in series. In this case, the voltage drop across each of the junctions would be one-half of the applied voltage, thus fitting to the I-V curve of a tunnelling junction would yield a barrier height which would be twice the work function of $\mathrm{Au}$. The fact that 


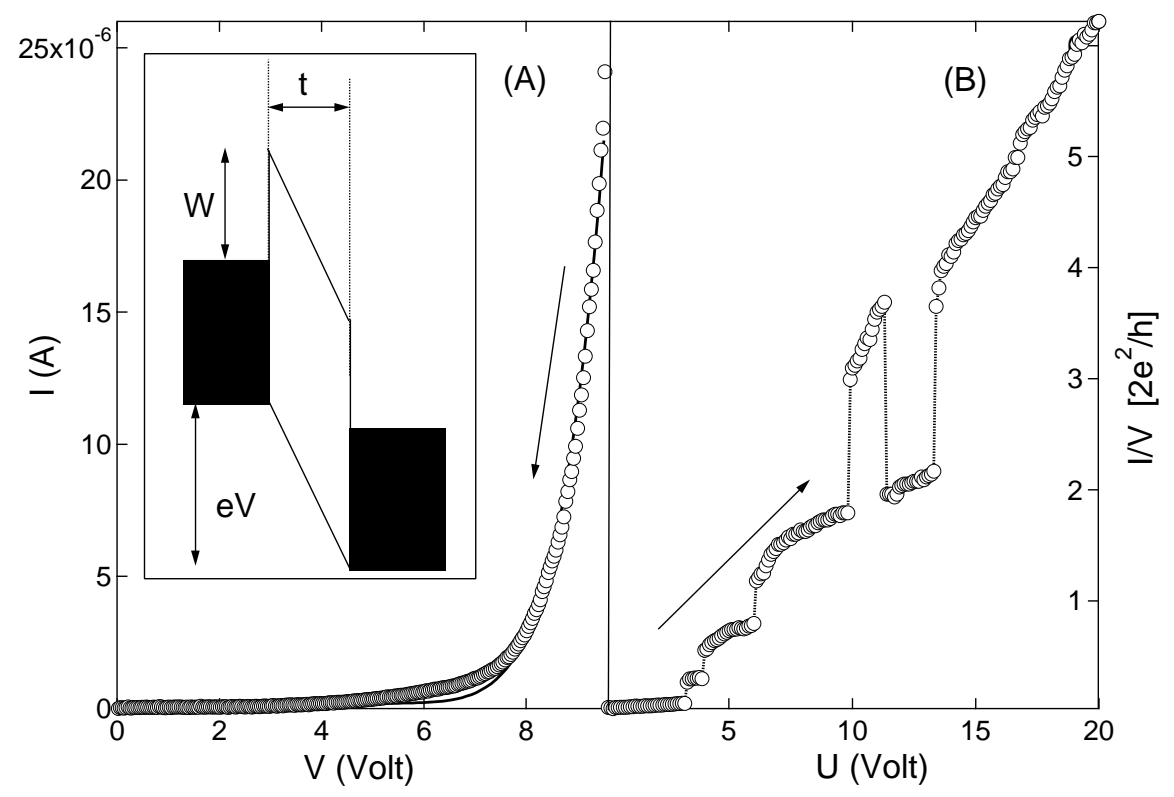

FIG. 4: A: Circles: I-V curve of a Au nano-junction. Line: fit to the field emission model. Inset: Schematic of the field emission model. $W$ is the tunnelling barrier height and $t$ is the barrier thickness. The best fit parameters: $W=5.8 \mathrm{eV}$ and $t=10.1 \AA$ A B: Discrete steps in conductance of order $2 e^{2} / h$ in a current limited Au nano-junction.

the best fit parameter for the barrier height is only slightly larger than the work-function of $\mathrm{Au}$ indicates that the lead resistance is much smaller than the resistance $V / I$ of the junction at $10 \mathrm{~V}$ bias voltage, e.g. $R_{\text {lead }} \ll 250 k \Omega$ or $G_{\text {lead }} \gg 0.1 e^{2} / h$.

It is striking that tunnelling contacts survive at $10 \mathrm{~V}$ bias voltage, since a conventional tunnelling junction with a similar barrier thickness would typically suffer an electric break down at $10 \mathrm{~V}$. We explain the stability of our atomic-scale gaps at $10 \mathrm{~V}$ with a dynamical equilibrium between two opposing atom migration processes. ${ }^{15}$ At $10 \mathrm{~V}$, the surface diffusion is opposed by electromigration (which increases the gap between the two films).

\section{Atomic-Scale Contacts}

After reducing the bias voltage quickly to zero as described above, we introduce a serial resistor $R_{S}=20 k \Omega$ and start increasing $U$ at a rate of $1 \mathrm{~V} / \mathrm{s}$. The serial resistor limits the current flowing through the junction, thereby limiting electromigration. Consequently, the conductance can exceed $e^{2} / h$. 
At a bias voltage of $U \sim 4 V$, conductance of the device begins to increase in discrete steps as a function of $U !^{10}$ An example is shown in Fig. 44. The step size is of order $0.2-2 e^{2} / h$, suggesting that the junction contains atomic-scale contacts. We have recently confirmed these discrete conductance steps at series resistance $R_{S}=100 \mathrm{k} \Omega$, showing that the conductance steps are intrinsic to the junction and not biased by our choice of $R_{S}$.

In addition to these discrete steps, the conductance changes continuously as a function of $U$, suggesting that there is a distributed contribution to the resistance of the junction, from the leads. In Fig. 3 C and D we show the junctions from Fig. 3 A and D, respectively, while inside the SEM, after the conductance was increased to $\approx 2 e^{2} / h$ and $\approx 6 e^{2} / h$, respectively. One notices that the length of the junctions increases with conductance. We observe that the conductance is roughly proportional to the length. The conductance per unit length is $G / L \approx 600 \mathrm{~S} / \mathrm{m}$. Among different samples, $G / L$ fluctuates by about a factor of two.

Thus, the increase in $G$ arises from the addition of $\mathrm{Au}$ into the nano-junction. Notice that the gap in the junction in Fig. 3-D remains well defined. We thus arrive at a model for the nano-junction sketched in Fig. 1. Reservoirs $R_{1}$ and $R_{2}$ are bulk Au films, which are good conductors with sheet resistance of $\approx 5 \Omega . C_{1}$ and $C_{2}$ are atomic-scale contacts responsible for conductance quantization. Finally, $L_{1}$ and $L_{2}$ are the disordered leads generated by the atom migration processes. From the images in Fig. 3. we obtain that the size of the leads ( $D$ in Fig. 11) is approximately 50nm.

Using Ohms laws, the conductance of the junction can be written as

$$
G=\sum_{i} \frac{1}{1 / G^{i}+1 / G_{L_{1}}^{i}+1 / G_{L_{1}}^{i}}
$$

where $G^{i}$ refers to the conductance of an atomic scale contact in the gap, and $G_{L_{1,2}}^{i}$ are the conductances between the contacts and the reservoirs. As the junction dimensions increase, $G_{L_{1,2}}^{i}$ changes continuously and $G^{i}$ changes in discrete steps of order $e^{2} / h$.

Because the continuous change in $G$ in Fig. 4 $4 \mathrm{~B}$ is comparable to the discrete steps in $G$, it follows that the lead resistance is comparable with the resistance of the atomic-scale contacts. To obtain the resistivity of the leads, we need to know the cross-section of these protrusions. Unfortunately we can not obtain this information through SEM-imaging. If we assume that the cross-section of the protrusion has the thickness of $50 \mathrm{~nm}$, which is comparable to the film thickness, we obtain $\rho \approx 1.7 \cdot 10^{5} \mu \Omega \mathrm{cm}$.

The resistivity is much larger than the maximum metallic resistivity of $\sim 200 \mu \Omega \mathrm{cm}, 17$ 
which shows that the the leads are highly disordered. The disorder is explained by the intermixing of the impurities into the leads and grain boundaries. ${ }^{10}$ In the device in Fig. 3 . D (and many other devices), the leads appear completely uniform down to the imaging resolution $(3 \mathrm{~nm})$. We still expect the leads to be granular, with grain diameter (d) smaller than $3 \mathrm{~nm}$, because Au does not form alloys with water (or other impurities such as $\mathrm{O}_{2}$ and $\mathrm{CO}_{2}$ that are present at $10^{-6}$ Torr background pressure).

In three-dimensional granular systems, the resistance between the grains $\left(R_{g}\right)$ and the resistivity are related as $\rho \sim R_{g} d$ and it is known that granular systems in 3D exhibit a metal-insulator transition as a function of $R_{g}$. 18 Theoretically, it has been predicted that

the transition occurs at $R_{g}=R_{g}^{C} \sim 19 R_{Q} / \ln \left(E_{C} / \delta\right)$, where $E_{C}$ is the charging energy of the grain and $\delta$ is the level spacing inside the grain. ${ }^{19.20}$ In our case, the grain diameter is less than $3 \mathrm{~nm}$ and $\rho \approx 10^{5} \mu \Omega \mathrm{cm}$, and we estimate $R_{g}>12 h / e^{2}$ and $R_{g}^{C} \sim 5 h / e^{2}$. Thus, we expect that the electronic states in the leads are strongly localized.

If the localization length is smaller than the dimensions of the leads, then the lead resistance at low temperature becomes much larger than the resistance of the atomic-scale contacts. The temperature dependence of the resistance becomes significant at temperatures well below 300K, whereas conductance quantization in Au is easily observed at room temperature. This explains the difference between room-temperature and low-temperature properties of nano-junctions.

\section{ZERO BIAS ANOMALIES AND COULOMB BLOCKADE}

Electron transport measurements at low temperatures were carried out using a dilution refrigerator with a base temperature of $0.015 K$. The bias voltage, applied to the sample, was the sum of a DC-voltage $V$ and an $\mathrm{AC}$ voltage with peak-to-peak amplitude $<10 \mu V$ and frequency $<100 \mathrm{~Hz}$. A current amplifier measured the current, while lock-in detection from the amplifier output obtained the differential conductance. The devices were shielded at $T=0.015 K$ by a Faraday cage and home made radiation filters. The base electron temperature was $\sim 0.05 K$.

Transport properties of our junctions changed dramatically when the temperature was reduced from $300 \mathrm{~K}$ to $0.015 \mathrm{~K}$. At $300 \mathrm{~K}$, the junctions were Ohmic and displayed conductance quantization effects. At low temperatures, however, the junctions showed significant 
suppression near zero-bias voltage.

Devices with $G(300 K)<e^{2} / h$ display Coulomb-blockade at $T=0.015 K$. The Coulomb blockade has been attributed to single electron charging effects in the grains inside the leads. 10

Devices with $G(300 K)>2 e^{2} / h$ do not display Coulomb blockade at $0.015 K$. Instead, the conductance versus voltage at $T=0.015 K$ displays a ZBA. The ZBAs were interpreted as the Coulomb-Blockade effect in the strong tunnelling regime. ${ }^{10}$

\section{A. Microscopic Origin of the Charging Effects and ZBAs}

We have found that the Coulomb Blockade in our Au junctions is not restricted to singleelectron charging effects in the grains in the leads. In fact, the necessary condition to observe Coulomb-Blockade in our devices is that the leads be highly resistive, regardless of whether the disorder in the leads is granular or homogeneous. For example, if we compare Figs. 3 C and D, we observe that the leads of the nano-junction in Fig. 3. C have well distinguished grains, whereas the leads of the nano-junction in Fig. 3 D are completely uniform. Despite these differences, the $\mathrm{I}-\mathrm{V}$ curves of these samples at $T=0.015 K$ are very similar.

We are led to the conclusion that the Coulomb-Blockade and ZBAs at low temperature arise from localization of electronic states in the leads, which could either be due to localization of electrons within one or a few grains (Fig. 3. C), or due to localization over a region containing a large number of grains that are too small to observe by the SEM (Fig. 3 D). In the Coulomb-blockade regime, electron transport is sequential and takes place via a puddle of electrons in the leads, which is sketched in Fig. 11B. In sec. IV] we show that the size of the puddle of electrons in Fig. 3.D is comparable to the dimensions of the leads.

Coulomb Blockade in distributed systems has been studied in disordered $\operatorname{In} O_{x}$ mesoscopic wires. ${ }^{21}$ Transport properties of these wires exhibited single electron charging effects at low temperature, very similar to those in single-electron transistors (SET). However, these wires

had no apparent tunnelling barriers. The single electron charging effects were observed if the localization length had been smaller than the sample size. It was suggested that the size of the puddles was comparable to the localization length, but it remained unclear what the junctions were and what formed the puddles of electrons. 


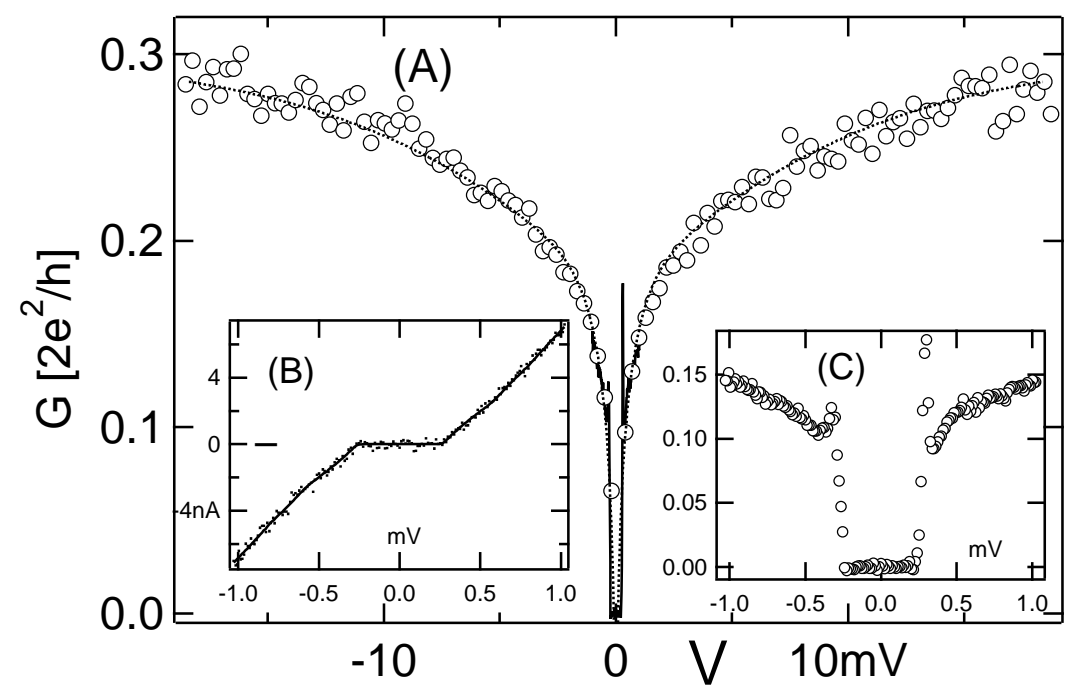

FIG. 5: A: Differential conductance versus bias voltage of a $\mathrm{Au}$ nano-junction at $T=0.015 K$. B: Current versus voltage at $T=0.015 K$ of the nano-junction in a narrow voltage range. C: Differential conductance versus voltage of the nano-junction at $T=0.015 K$ in a narrow voltage range.

\section{B. Effective Charging Energy}

Among devices, the charging energy rapidly decreases as a function of $G(300 K)$. Fig. 5 A shows the conductance versus bias voltage at $T=0.015 K$ in a device with $G(300 K)=$ $0.7 e^{2} / h$. This device belongs to a group of borderline devices in which the Coulomb blockade is just resolved at $T=0.015 K$.

The borderline devices are characterized by two voltage scales. If the voltage range is large, e.g. $[-20 \mathrm{mV}, 20 \mathrm{mV}]$ in Fig. 5-A, the curve resembles ZBAs of high conductance devices. Thus, in this voltage range we fit the curve to the model of electron tunnelling through a single-electron transistor in the strong tunnelling regime ${ }^{13}$ This leads to the parameter estimates $C_{1}+C_{2}=20.8 a F, R_{1}=2.7 k \Omega$, and $R_{2}=34.3 \mathrm{k} \Omega$, where $C_{1}$ and $C_{2}$ are the bare capacitances between the puddle and the reservoirs, and $R_{1}$ and $R_{2}$ are the bare resistances between the puddle and the reservoirs. The corresponding bare charging energy is $e^{2} / 2\left(C_{1}+C_{2}\right)=3.8 \mathrm{meV}$. The best fit is shown by the dotted line in Fig. 5 -A.

The conductance in Fig. 5-A approaches zero at a nonzero zero-bias voltage. Fig. 5.C 
zooms in to Fig. 5. A around zero bias voltage. The corresponding I-V curve is shown in Fig. 5-B. The gap in the I-V curve represents Coulomb-Blockade. By fitting the low bias voltage I-V curves to the Orthodox theory of single-electron tunnelling, 11,12 we estimate $\tilde{C}_{1}+\tilde{C}_{2}=442 a F, \tilde{R}_{1}=34 k \Omega$, and $\tilde{R}_{2}=65 k \Omega$ for the capacitances and the resistances between the puddle and the reservoirs. The fit is shown by the line in Fig. 5.B. The corresponding charging energy is $\tilde{E}_{C}=e^{2} / 2\left(\tilde{C}_{1}+\tilde{C}_{2}\right)=0.18 \mathrm{meV}$, a factor of 21 smaller than the bare charging energy estimated above.

Theoretically, it has been predicted that Coulomb-Blockade persists in any diffusive conductor, even if the resistances between the conductor and the reservoirs is much larger than the resistance quantum. ${ }^{14.22}$ The persistence of charging effects in a single electron transistor in strong coupling to the leads has been demonstrated experimentally: ${ }^{23,24}$ It has been predicted that the effective charging energy is given as

$$
\tilde{E}_{C}=E_{C} e^{-\alpha \frac{G}{G_{0}}}
$$

where $G=G_{1}+G_{2}$ is the sum of the conductances between the conductor and the reservoirs, $G_{0}=2 e^{2} / h$, and, finally, $\alpha$ is a constant of order one ${ }^{14,22}$

In our samples, $E_{C}$ and $\tilde{E}_{C}$ are interpreted as the bare and effective charging energy, respectively. With $\alpha \approx 0.6$, they are in rough agreement with Eq. 2 ,

\section{CAPACITANCE FLUCTUATIONS}

In conventional single-electron transistors, the charging energy is independent of the applied magnetic field. In contrast, we find that the effective charging energy of our nanojunctions exhibits strong magnetic field dependence.

Fig. 6] displays a gray-scale image of the conductance versus bias voltage and the applied magnetic field in the sample with the I-V curves shown in Fig. 5. The magnetic field is parallel to the slit. The threshold voltage for Coulomb Blockade (the gap) exhibits a strong non-monotonic dependence - fluctuations - with the magnetic field. Around the field of $2 \mathrm{~T}$, the gap approaches zero, and around the field of $11 \mathrm{~T}$, the gap is at maximum. The dependence is reproducible when the measurements are repeated. The amplitude of the gap fluctuations is comparable to the average gap.

The characteristic magnetic field scale of the gap fluctuations $\left(B_{C}\right)$ is given by the typical 


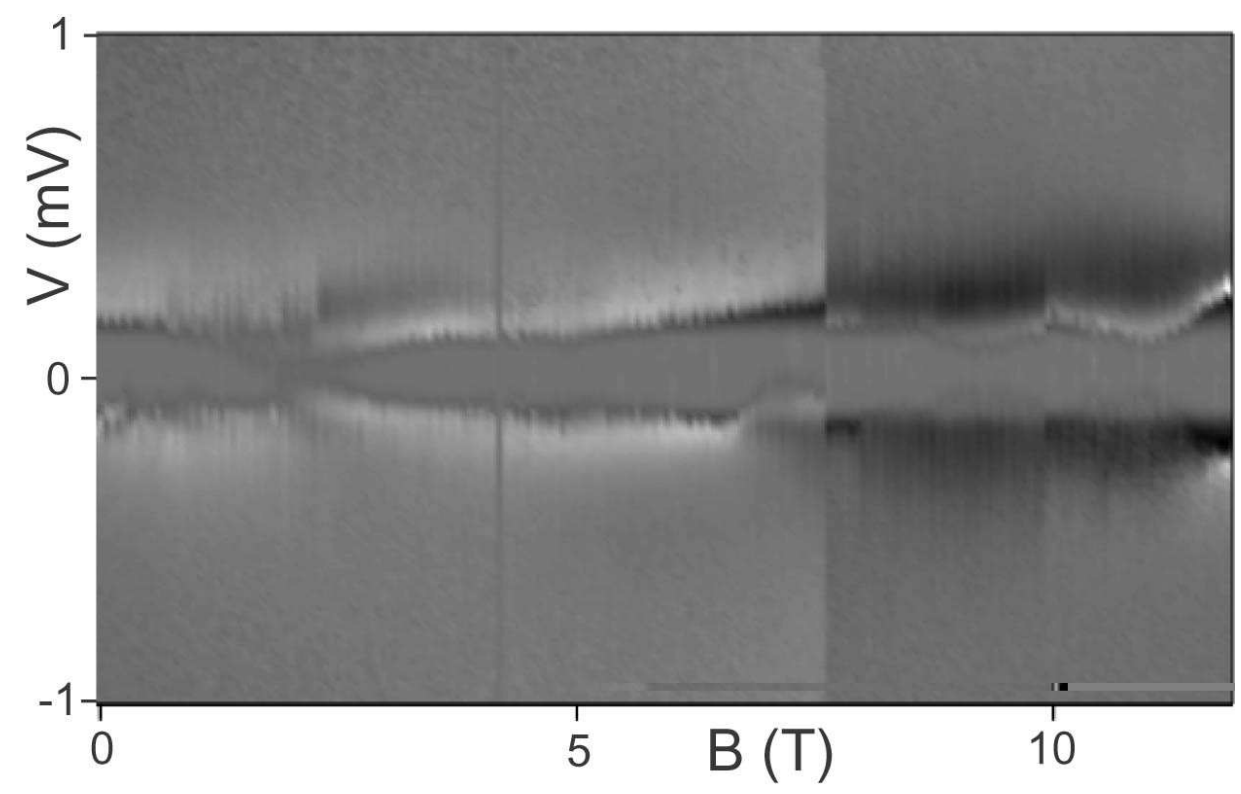

FIG. 6: Conductance (gray) versus magnetic field and bias voltage of the nano-junction at $T=$ $0.015 K$.

period of the fluctuations. We resolve less than a full period in our magnetic field range, suggesting $B_{C} \approx 18 T$. We have confirmed the gap fluctuations in 4 additional samples with similar effective charging energies. The value of $B_{C}$ is reproducible within a factor of 2 among these samples.

We now show that the fluctuations in the gap represent charging energy fluctuations (or capacitance fluctuations). To this end, we examine the gate voltage dependence of the conductance, as a function of the applied magnetic field. Figure [7 displays conductance versus gate voltage and bias voltage at magnetic fields of 0T, 4T, $8 \mathrm{~T}$, and $12 \mathrm{~T}$, in a different sample (the previous sample did not have a gate). The fabrication of the gate has been described in Ref. ${ }^{10}$.

Fig. 77-A resembles "diamond diagrams" of conductance versus gate voltage and bias voltage of quantum dots. ${ }^{25}$ The strong dependence of the gap on gate voltage proves that the gap is caused by the Coulomb-Blockade. In particular, at certain gate voltages, indicated by the groups of four lines that cross at a point along the $\mathrm{V}=0$ axis, the gap approaches zero. These points will be referred to as points where the diamonds close, and the conductance at these points will refer to the peak conductance $\left(G_{\text {peak }}\right)$. The valley conductance $G_{\text {valley }}$ is defined as the conductance at $V=0$ and at a gate voltage where the gap is at maximum. 


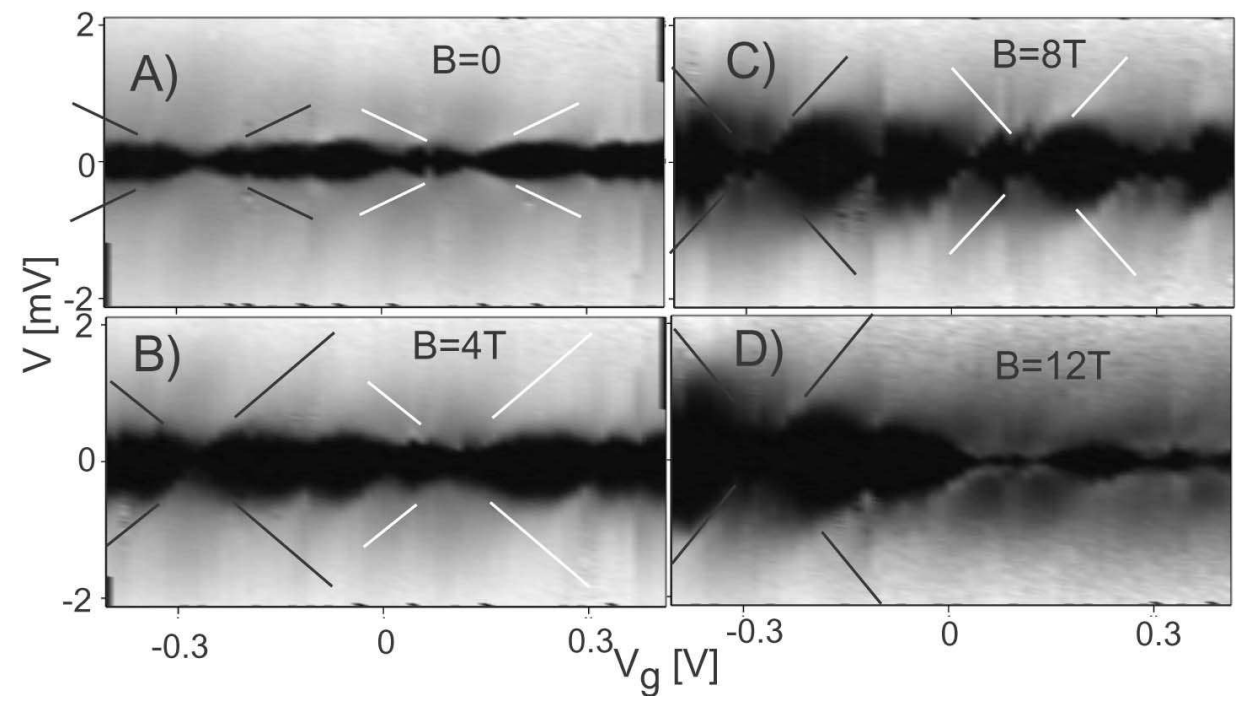

FIG. 7: A-D: Conductance of a Au nano-junction (gray) versus gate voltage and bias voltage at four magnetic fields at $T=0.015 K$.

There are significant differences between the diamonds in Fig. 7 and the diamonds of conventional single-electron transistors. First, the gate voltage dependence of the gap in Fig. 7 is not periodic. We examined the gate voltage dependence in the range of gate

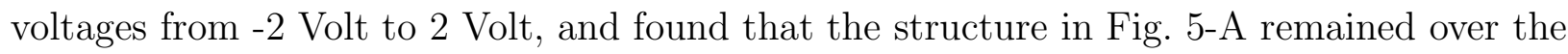
extended voltage range. The structure in Fig. 5. A is quasiperiodic, in that the slopes of the diamond's edges, near the points where diamonds close, are the same (i. e. the lines in the black and the white groups in Fig. 5 -A have the same slopes).

Discontinuities in conductance, as a function of gate voltage, cause the absence of periodicity in Fig. [7 A. When the gate voltage sweeps are repeated, conductance discontinuities are reproducible, and can be attributed to the shifts in the background charge induced by the changes in gate voltage. The leads are highly disordered, thus they may contain a large number of charge traps in the vicinity of the puddle responsible for Coulomb Blockade. The gate voltage can change the state of the charge trap, and causes a discontinuous shift in the background charge.

The second difference between Coulomb Blockade in our nano-junctions and that of conventional SETs is found in the conductance peak's temperature dependence. Fig. 8 shows $G_{\text {peak }}$ and $G_{\text {valley }}$ versus temperature. $G_{\text {peak }}$ decreases significantly with temperature even when $k_{B} T \ll \tilde{E}_{C}$. In contrast, with conventional SETs, $G_{\text {peak }}$ has a weak temperature dependence when $k_{B} T$ is much smaller than the charging energy. It appears that $G_{\text {peak }}$ 


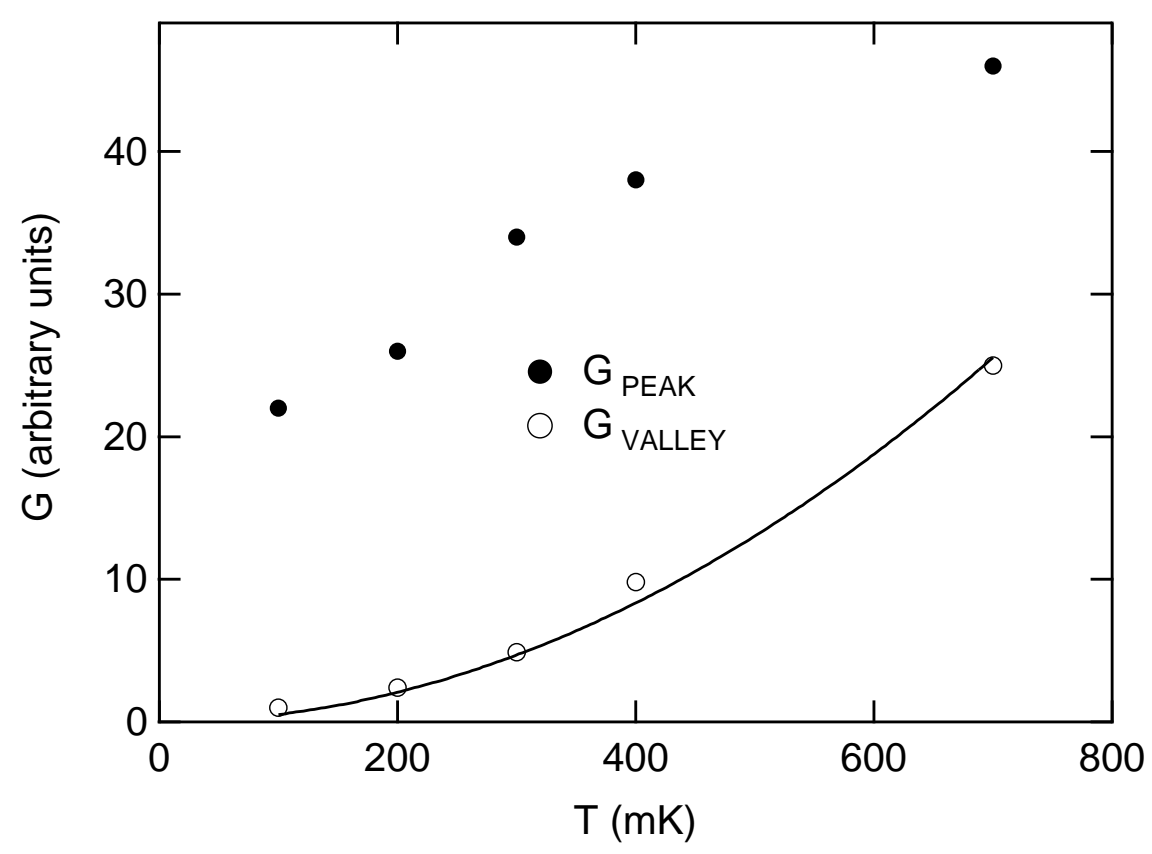

FIG. 8: Temperature dependence of the peak and the valley conductance. The line displays the best fit of the valley conductance to the quadratic temperature dependence.

approaches a nonzero value when $T \rightarrow 0$.

At low temperature $\left(k_{B} T \ll \tilde{E}_{C}\right)$, the valley conductance goes to zero as $G_{\text {valley }} \sim T^{2}$, as shown in Fig. 8 . The quadratic temperature dependence in the valleys demonstrates that electron transport in the valleys occurs through inelastic cotunnelling, $\stackrel{26}{=}$ which is possible only if the spacing between energy levels $(\delta)$ in the puddle of electrons is much smaller than $k_{B} T$. Assuming that the level spacing is given by $\delta \approx 1 /(N(0) V)$, where $N(0)$ is the density of states at the Fermi level of $\mathrm{Au}$, and $V$ is the volume of the puddle, we obtain that $V>(10 \mathrm{~nm})^{3}$. This suggests that the localization length is larger than $10 \mathrm{~nm}$.

At low magnetic fields $(\leq 8 T)$, we can trace the evolution of the diamonds with the magnetic field quite well, despite the discontinuities in the background charge. The points where diamonds close do not shift with magnetic field in this range. This implies that the capacitance between the puddle and the gate $\left(C_{g}\right)$ does not vary. Therefore, the geometry of the puddle does not change with magnetic field.

The key effect in Fig. [7 A-C is that it is the puddle's effective charging energy that changes strongly with magnetic field. From the Orthodox theory of Coulomb-Blockade, $\underline{\underline{12}}$ the slopes of the lines in Fig. 7 are $\pm e C_{g} /\left(2 \tilde{C}_{1}\right)$ and $\pm e C_{g} /\left(2 \tilde{C}_{2}\right)$, where $\tilde{C}_{1}$ and $\tilde{C}_{2}$ are the effective 
capacitances between the puddle and the reservoirs. It follows that $\tilde{C}_{1}$ and $\tilde{C}_{2}$ fluctuate with field. In particular, from Fig. [7] we obtain $\tilde{C}_{1}(4 T)=1.8 \tilde{C}_{1}(0), \tilde{C}_{1}(8 T)=2.3 \tilde{C}_{1}(0)$, and $\tilde{C}_{1}(12 T)=2.4 \tilde{C}_{1}(0)$.

Nazarov had predicted fluctuations of effective capacitance in coherent conductors in the regime of strong coupling to the reservoirs. ${ }^{14}$ With strong coupling, the Coulomb-Blockade survives in any coherent disordered conductor. In this regime, effective capacitance exhibits mesoscopic fluctuations as a function of the applied magnetic field. These fluctuations are analogous to universal conductance fluctuations. ${ }^{27}$

One way to understand capacitance fluctuations is to observe that the effective charging energy, $e^{2} / 2\left(\tilde{C}_{1}+\tilde{C}_{2}\right)$, exponentially depends on the conductance between the conductor and the reservoirs, see Eq. 2. Then, the universal conductance fluctuations induce fluctuations in $G_{1}+G_{2}$ with field, which leads to the fluctuations in the effective charging energy. Since the amplitude of conductance fluctuations in the diffusive regime is $\sim G_{Q}$, it follows that the amplitude of the charging energy fluctuations is comparable to the average charging energy, consistent with our data.

We expect that the characteristic magnetic field scale is given by the flux quantum $\left(\Phi_{0}=\right.$ $h / 2 e$ ) over the directed area of the puddle, $B_{C} \sim \Phi_{0} / L_{s}^{2}$, where $L_{S}$ is the diameter of the puddle (localization length). For $B_{C} \approx 18 T$, we obtain $L_{s} \approx 105 \mathrm{~nm}$, which is comparable to the dimensions of the leads $D \approx 50 \mathrm{~nm}$.

If the magnetic field approaches $12 \mathrm{~T}$, it becomes hard to trace the diamonds. In fact, at the field of $12 T$ (Fig. 7-D), the structure is no longer quasiperiodic. This suggests that when the magnetic field approaches $B_{C}$, conduction can no longer be described by sequential tunnelling via the same puddle of electrons. The strong-field regime is the subject of current research.

\section{COMPARISON WITH ELECTROPLATED NANO-JUNCTIONS}

Our introduction described strong ZBAs, observed in electroplated Au nano-junctions containing atomic-scale contacts. ${ }^{6.7}$ The bulk electroplated material was found not to undergo a strong localization transition at low temperatures. In addition, the ZBAs exhibited scaling with junction size that could not be easily explained in the localization framework. The scaling suggested that the ZBAs displayed a suppression in the density of states in the 
leads.

Note that only if the resistance of atomic-scale contacts is much larger than the lead resistance can the conductance of the contact be proportional to the density of states in the leads, as would be the case in conventional tunnelling junctions. ${ }^{28}$ Thus, a $\sim 100 \%$ suppression of the density of states in electroplated nano-junctions must be very local around the atomic-scale contact. If it were otherwise, the conductivity of the leads would be $\sim 100 \%$ reduced in a region much larger than the contact size, and the lead resistance would not be much smaller than the resistance of the contact.

In our devices, the localization length is comparable to the dimensions of the leads and the conductance is not proportional to the density of states. The ZBAs in our nano-junctions are caused by the Coulomb blockade on localized puddles of electrons inside the leads, analogous to Coulomb-Blockade in disordered $\operatorname{InO}_{x}$ wires ${ }^{21}$ The ZBAs are manifestations of Coulomb-Blockade on these puddles in the regime of strong-coupling to the reservoirs.

\section{CONCLUDING REMARKS}

Atomic-scale point contacts of Au between strongly disordered leads can have striking differences between their room-temperature and the low temperature properties. At room temperature the contacts exhibit conductance quantization and are Ohmic, at low temperatures the contacts exhibit Coulomb-Blockade or zero-bias anomalies. The differences between the room-temperature and the low temperature properties arise from the localization of electronic states in the leads. The temperature at which the resistance of the leads becomes significantly larger than the resistance of the contacts is much lower than the room temperature.

At low temperature, Coulomb-Blockade arises from puddles of electrons in the leads that form as a result of localization. One can distinguish between the bare charging energy and the effective charging energy of the puddles. The latter is found to be much smaller than the former, and it exhibits strong fluctuations with an applied magnetic field. The gate voltage effects of a magnetic field demonstrate that the effective capacitance between the puddle and the reservoirs fluctuates with the magnetic field, in agreement with theoretical predictions. 


\section{Acknowledgments}

This work was performed in part at the Cornell Nanofabrication Facility, (a member of the National Nanofabrication Users Network), which is supported by the NSF, under grant ECS-9731293, Cornell University and Industrial affiliates, and the Georgia-Tech electron

microscopy facility. This research is supported by the David and Lucile Packard Foundation grant 2000-13874 and the NSF grant DMR-0102960.

1 C. J. Muller, J. M. van Ruitenbeek, and L. J. de Jongh, Physica C 191, 485 (1992).

2 N. Agrait, A. L. Yeyati, and J. M. V. Ruitenbeek, Phys. Rep. 377, 81 (2003).

3 A. F. Morpurgo, C. M. Marcus, and D. B. Robinson, Appl Phys Lett 74, 2084 (1999).

4 C. Z. Li, H. X. He, A. B. A, J. S. Bunch, and N. J. Tao, Phys. Rev. A 76, 1333 (2000).

5 S. Boussaad and N. J. Tao, Appl Phys Lett 80, 2398 (2002).

6 L. H. Yu and D. Natelson, Appl Phys Lett 82, 2332 (2003).

7 L. H. Yu and D. Natelson, Phys. Rev. B 68, 113407 (2003).

8 H. Park, A. K. L. Lim, A. P. Alivisatos, J. Park, and P. L. McEuen, Appl. Phys. Lett. 75, 301 (1999).

9 J. Park, A. N. Pasupathy, J. I. Goldsmith, C. Chang, Y. Yaish, J. R. Petta, M. Rinkoski, J. P. Sethna, H. D. Abruna, P. L. McEuen, et al., Nature 417, 722 (2002).

10 A. Anaya, A. L. Korotkov, M. Bowman, J. Waddell, and D. Davidović, Journal of Applied Physics 93, 3501 (2003).

11 D. V. Averin and A. N. Korotkov, Journal of low temperature physics 80, 173 (1990).

12 D. V. Averin and K. K. Likharev, in Mesoscopic Phenomena in Solids, edited by B. L. Altshuler, P. L. Lee, and R. A. Webb (Elsevier and Amsterdam, 1991), p. 169.

13 D. S. Golubev, J. Konig, H. Schoeller, G. Schon, and A. D. Zaikin, Phys. Rev. B 56, 15782 (1997).

14 Y. V. Nazarov, Phys. Rev. Lett. 82, 1245 (1999).

15 A. L. Korotkov, M. Bowman, H. J. McGuinness, and D. Davidovic, Nanotechnology 14, 42 (2003).

16 T.M.Mayer, J.E.Hueston, G.E.Franklin, A.A.Erchak, and Michalske, Jour. Appl. Phys. 85, 8170 
(1999).

17 Y. Imry, Introduction to mesoscopic physics (Oxford University Press, 1997).

18 K. B. Efetov and A. Tschersich, Phys. Rev. B 67, 174205 (2003).

19 I. S. Beloborodov, K. B. Efetov, A. Altland, and F. W. J. Hekking, Phys. Rev. B 63, 115109 (2001).

20 I. S. Beloborodov, K. B. Efetov, A. V. Lopatin, and V. M. Vinokur, http://xxx.lanl.gov/abs/cond-mat/0304448 (????).

21 V. Chandrasekhar, Z. Ovadyahu, and R. A. Webb, Phys. Rev. Lett 67, 2862 (1991).

22 S. V. Panyukov and A. D. Zaikin, Phys. Rev. Lett. 67, 3168 (1991).

23 D. Chouvaev, L. S. Kuzmin, D. S. Golubev, and A. D. Zaikin, Phys. Rev. B 59, 10599 (1999).

24 P. Joyez, V. Bouchiat, D. Esteve, C. Urbina, and M. H. Devoret, Phys. Rev. Lett. 79, 1349 (1997).

25 L. P. Kouwenhoven, C. Marcus, P. McEuen, S. Tarucha, R. Westervelt, and N. Wingreen, in Mesoscopic Electron Transport, edited by L. P. Kouwenhoven, L. L. Sohn, and G. Schon (Elsevier and Amsterdam, 1997), p. 549.

26 D. V. Averin and Y. V. Nazarov (Kluwer Academic/Plenum Publishers, 1991).

27 S. Washburn and R. A. Webb, Rep. Prog. Phys. 55, 1311 (1992).

28 B. L. Altshuler and A. G. Aronov, in Electron-Electron Interactions in Disordered Systems, edited by A. L. Efros and M. Pollak (Elsevier and Amsterdam, 1985). 